\title{
An Improved Lumped Element Nonlinear Circuit Model for a Circular CMUT Cell
}

\author{
Hayrettin Köymen, Senior Member, IEEE, Abdullah Atalar, Fellow, IEEE, Elif Aydoğdu, \\ Coşkun Kocabaş, H. Kağan Oğuz, Student Member, IEEE, Selim Olçum, Member, IEEE,
} Alper Ozgurluk, Student Member, IEEE, and Asli Ünlügedik

\begin{abstract}
This paper describes a correction and an extension in the previously published large signal equivalent circuit model for a circular capacitive micromachined ultrasonic transducer (CMUT) cell. The force model is rederived so that the energy and power is preserved in the equivalent circuit model. The model is able to predict the entire behavior of CMUT until the membrane touches the substrate. Many intrinsic properties of the CMUT cell, such as the collapse condition, collapse voltage, the voltage-displacement interrelation and the force equilibrium before and after collapse voltage in the presence of external static force, are obtained as a direct consequence of the model. The small signal equivalent circuit for any bias condition is obtained from the large signal model. The model can be implemented in circuit simulation tools and model predictions are in excellent agreement with finite element method simulations.
\end{abstract}

\section{INTRODUCTION}

$\mathrm{T}$ HE need for an accurate lumped element equivalent circuit model for capacitive micromachined ultrasonic transducers (CMUTs) has been extensively discussed [1]-[7]. The finite element method (FEM) is a powerful technique for the analysis of CMUTs, when the number of CMUT cells in an array is low [8]-[11]. The CMUT operation can be accurately simulated and information on the nonlinear effects, medium loading, cross talk, and the effect of the higher-order harmonics can be obtained.

An iterative approach must be adopted, however, to design CMUTs using FEM. This approach is very computationally intensive and can be time consuming. Getting results with FEM analysis for arrays which contain a large number of CMUT cells is practically impossible. However, realization of arrays comprising a large number of cells at low cost is one of the fundamental advantages of CMUT technology [12]-[15].

Design and analysis of CMUTs using lumped element equivalent circuit requires the knowledge of radiation

Manuscript received December 28, 2011; accepted May 20, 2012. This work was supported by the Scientific and Technological Research Council of Turkey (TUBITAK) under project grant 110E216. A. Atalar acknowledges the support of the Turkish Academy of Sciences (TUBA).

H. Köymen, A. Atalar, E. Aydoğdu, H. K. Oğuz, A. Ozgurluk, and A. Ünlügedik are with the Electrical and Electronics Engineering Department, Bilkent University, Ankara, Turkey (e-mail: koymen@ee.bilkent. edu.tr).

C. Kocabaş is with the Physics Department, Bilkent University, Ankara, Turkey.

S. Olçum is with the Department of Biological Engineering, Massachusetts Institute of Technology, Cambridge, MA.

DOI http://dx.doi.org/10.1109/TUFFC.2012.2383 impedance. The radiation interface must be accurately included in the model. The equivalent circuits of single CMUT cells can then be used to model arrays by appropriately terminating each cell with respective impedance. Recently, there has been a significant improvement on this topic for both single cells and arrays [16]-[18].

In this paper, we present an improvement on a previously reported large signal equivalent circuit model [1]. Because a CMUT is a distributed system, lumped variables are chosen to represent distributed quantities such as force or membrane velocity. In the earlier model, in which the rms membrane velocity, $v_{\text {rms }}$, is defined over the velocity profile spatially across the membrane surface, and the total force generated by the electric field, $F_{\text {tot }}$, were employed as the through and across variables, respectively. The model contains a nonlinear force term, $F_{\text {tot }}$, and a linear mechanical section, in which the section elements are defined according to the chosen through variable. To obtain $F_{\text {tot }}$ in [1], we used a generalization of Mason's [19] approach for a deflected membrane. The force distribution on the membrane surface was evaluated first and then it is integrated across the membrane surface to find $F_{\text {tot }}$. The through and across variables in the model are not connected through an energy relation in this approach. We have determined that the model obtained by this approach is not consistent, as far as preserving the energy of the system is concerned, although it is in good agreement with FEM results compared with other models. The differences between the model and FEM results were given in [1]. Mason's model suffers the same shortcoming.

In this paper, we present a force definition that is consistent with the choice of the through variable by linking them directly. We discuss the dependence of the equivalent circuit on the choice of through variable employed in the linear mechanical section and provide the results for three possible velocity definitions. We quantify the collapse voltage analytically as a function of the external static force, such as atmospheric pressure, and the cell parameters gap height, clamp capacitance, and membrane compliance, as a direct consequence of the model. The model is for the uncollapsed mode of operation: it very accurately predicts the behavior of the CMUT until the membrane touches the substrate. The force equilibrium on the membrane before and beyond the collapsing displacement is derived, again in terms of model parameters. The relevant equations for analytical design and implementation in circuit simulators are given. The radiation interface is completely modeled, and dependence of the radiation medium variables and 


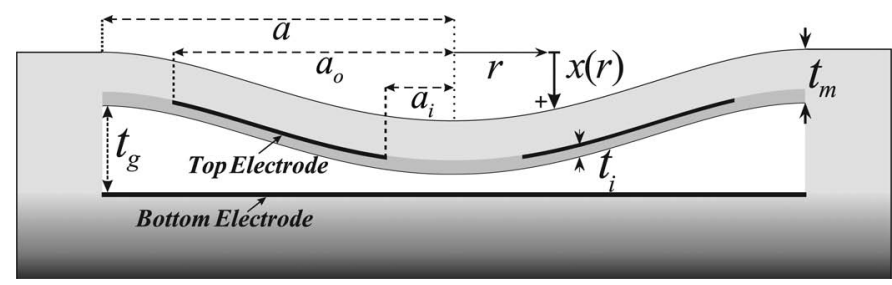

Fig. 1. Two-dimensional view and the dimensional parameters of the circular capacitive micromachined ultrasonic transducer (CMUT) geometry.

circuit variables of the mechanical section is discussed and clarified. Because most receivers are operated under small signal conditions, a linear small signal equivalent circuit is derived from the large signal model and presented.

\section{Lumped Element Equivalent Circuit Model for CMUT}

\section{A. Defining the Through and Across Variables}

The basic geometry of a circular CMUT with a partial electrode is given in Fig. 1. The displacement profile for thin clamped plates or membranes obtained using plate theory [20], [21], when depressed by uniform pressure, is

$$
x(r, t)=x_{\mathrm{P}}(t)\left(1-\frac{r^{2}}{a^{2}}\right)^{2} \quad \text { for } r \leq a,
$$

where $a$ is the radius of the aperture, $r$ is the radial position, and $x_{\mathrm{P}}$ is the displacement at the center of the membrane; positive displacement is toward the bottom electrode. ${ }^{1}$ It is shown that CMUTs with full electrodes, with thin plate membranes, have the same profile [1]. The capacitance, $\delta C(r, t)$, of a concentric narrow ring on the membrane of radius $r$ and width $d r$ can be expressed as

$$
\delta C(r, t)=\frac{\varepsilon_{0} 2 \pi r \mathrm{~d} r}{t_{\mathrm{ge}}-x(r, t)}=\frac{\varepsilon_{0} 2 \pi r \mathrm{~d} r}{t_{\mathrm{ge}}-x_{\mathrm{P}}(t)\left(1-\frac{r^{2}}{a^{2}}\right)^{2}},
$$

where $\varepsilon_{0}$ is the permittivity of the gap and $t_{\mathrm{ge}}=t_{\mathrm{g}}+$ $t_{\mathrm{i}} / \varepsilon_{\mathrm{r}}$ is the effective gap height. Here, $t_{\mathrm{i}}$ and $t_{\mathrm{g}}$ are the thicknesses of the insulating layer and the vacuum gap height, respectively, and $\varepsilon_{\mathrm{r}}$ is the relative permittivity of the insulating material. The capacitance, $C(t)$, of the deflected membrane with a partial electrode of inner radius $a_{\mathrm{i}}$ and outer radius $a_{\mathrm{o}}$ can be found by an integration:

$$
C(t)=\int_{a_{\mathrm{i}}}^{a_{\mathrm{o}}} \delta C(r, t)=C_{0} g\left(\frac{x_{\mathrm{P}}(t)}{t_{\mathrm{ge}}}\right),
$$

where the function $g(\cdot)$ is defined by

\footnotetext{
${ }^{1}$ Throughout the paper, the first subscripts $\mathrm{R}, \mathrm{A}$, and $\mathrm{P}$ of mechanical variables refer to rms, average, and peak quantities, respectively.
}

$$
g(u)=\frac{\tanh ^{-1}\left(K_{\mathrm{i}} \sqrt{u}\right)-\tanh ^{-1}\left(K_{\mathrm{o}} \sqrt{u}\right)}{\sqrt{u}},
$$

where $K_{\mathrm{i}}=\left(1-a_{\mathrm{i}}^{2} / a^{2}\right), K_{\mathrm{o}}=\left(1-a_{\mathrm{o}}^{2} / a^{2}\right)$, and $C_{0}=$ $\varepsilon_{0} \pi a^{2} / t_{\mathrm{ge}}$.

If a voltage $V(t)$ is applied across the terminals, the instantaneous energy stored on the capacitance is given by $E(t)=1 / 2 C(t) V^{2}(t)$.

Suppose we choose the rms membrane velocity defined by

$$
v_{\mathrm{R}}(t)=\frac{\mathrm{d} x_{\mathrm{R}}(t)}{\mathrm{d} t}=\frac{\mathrm{d}}{\mathrm{d} t} \sqrt{\frac{1}{\pi a^{2}} \int_{0}^{a} 2 \pi r x^{2}(r, t) \mathrm{d} r}
$$

as the through variable of the equivalent circuit, which is defined in [22] as the spatial rms velocity. For the membrane profile in (1), we have $x_{\mathrm{R}}(t)=x_{\mathrm{P}}(t) / \sqrt{5}$. To preserve the energy, the corresponding across variable for force, $f_{\mathrm{R}}(t)$, should be written as

$$
\begin{gathered}
f_{\mathrm{R}}(t)=\frac{\partial E(t)}{\partial x_{\mathrm{R}}}=\sqrt{5} \frac{\partial E(t)}{\partial x_{\mathrm{P}}} \\
f_{\mathrm{R}}(t)=\sqrt{5} \frac{C_{0} V^{2}(t)}{2 t_{\mathrm{ge}}} g^{\prime}\left(\frac{x_{\mathrm{P}}(t)}{t_{\mathrm{ge}}}\right),
\end{gathered}
$$

where

$$
g^{\prime}(u)=\frac{1}{2 u}\left(\frac{K_{\mathrm{i}}}{1-K_{\mathrm{i}}^{2} u}-\frac{K_{\mathrm{o}}}{1-K_{\mathrm{o}}^{2} u}-g(u)\right)
$$

We also need the second derivative of (4) in this work, which is

$$
g^{\prime \prime}(u)=\frac{1}{2 u}\left(\frac{K_{\mathrm{i}}^{3}}{\left(1-K_{\mathrm{i}}^{2} u\right)^{2}}-\frac{K_{\mathrm{o}}^{3}}{\left(1-K_{\mathrm{o}}^{2} u\right)^{2}}-3 g^{\prime}(u)\right) .
$$

For $a_{\mathrm{i}} / a<0.25$ and for $a_{\mathrm{o}} / a \geq 0.8$, the displacement profile agrees well with the assumed profile and the material presented in this paper is applicable to such CMUTs. The profile deviates from (1) for other choices of $a_{\mathrm{i}}$ and $a_{\mathrm{o}}$ and the accuracy of the model deteriorates; however, the model predictions still provide good guidance for design.

For CMUTs with full electrodes (4), (8), (9), and (7) simplify to

$$
\begin{aligned}
g(u) & =\frac{\tanh ^{-1}(\sqrt{u})}{\sqrt{u}} \\
g^{\prime}(u) & =\frac{1}{2 u}\left(\frac{1}{1-u}-g(u)\right) \\
g^{\prime \prime}(u) & =\frac{1}{2 u}\left(\frac{1}{(1-u)^{2}}-3 g^{\prime}(u)\right)
\end{aligned}
$$

$$
f_{\mathrm{R}}(t)=\sqrt{5} \frac{C_{0} V^{2}(t)}{4 x_{\mathrm{P}}(t)}\left[\frac{t_{\mathrm{ge}}}{t_{\mathrm{ge}}-x_{\mathrm{P}}(t)}-\frac{\tanh ^{-1}\left(\sqrt{\frac{x_{\mathrm{P}}(t)}{t_{\mathrm{ge}}}}\right)}{\sqrt{\frac{x_{\mathrm{P}}(t)}{t_{\mathrm{ge}}}}}\right] .
$$


The series expansion of $g(u)$ around $u=0$ is

$$
\begin{aligned}
g(u)= & \left(K_{\mathrm{i}}+\frac{K_{\mathrm{i}}^{3}}{3} u+\frac{K_{\mathrm{i}}^{5}}{5} u^{2}+\frac{K_{\mathrm{i}}^{7}}{7} u^{3}\right) \\
& -\left(K_{\mathrm{o}}+\frac{K_{\mathrm{o}}^{3}}{3} u+\frac{K_{\mathrm{o}}^{5}}{5} u^{2}+\frac{K_{\mathrm{o}}^{7}}{7} u^{3}\right),
\end{aligned}
$$

from which its derivatives around $u=0$ can also be calculated. These are useful in circuit simulator applications in which $u \ll 1$.

The force in (11) is not the same as the total force on the membrane, $F_{\text {tot }}$, given in [1] as the across variable found using Mason's approach:

$$
F_{\mathrm{tot}}(t)=\frac{C_{0} V^{2}(t)}{4 t_{\mathrm{ge}}}\left[\frac{t_{\mathrm{ge}}}{t_{\mathrm{ge}}-x_{\mathrm{P}}(t)}+\frac{\tanh ^{-1}\left(\sqrt{\frac{x_{\mathrm{P}}(t)}{t_{\mathrm{ge}}}}\right)}{\sqrt{\frac{x_{\mathrm{P}}(t)}{t_{\mathrm{ge}}}}}\right]
$$

Fig. 2 is a comparison of these two force values as a function of $x_{\mathrm{P}} / t_{\mathrm{ge}}$. In [1, Eq. (10)], if the derivative had been taken with respect to $x_{\mathrm{P}}$, similar to the approach in [2], rather than $x$, there would have been an additional (1 $\left.r^{2} / a^{2}\right)^{2}$ term inside the integral and the two results would have been identical.

\section{B. Large Signal Equivalent Circuit}

The circuit variables on the electrical side can be found by considering the time rate of change of the instantaneous charge, $Q(t)=C(t) V(t)$, on the CMUT capacitance:

$$
\frac{\partial Q(t)}{\partial t}=C(t) \frac{\partial V(t)}{\partial t}+\frac{\partial C(t)}{\partial t} V(t)=i_{\mathrm{Cap}}(t)+i_{\mathrm{V}}(t)
$$

similar to the notation in [1]. Hence, the current components are

$$
i_{\mathrm{Cap}}(t)=C(t) \frac{\mathrm{d} V(t)}{\mathrm{d} t}=C_{0} \frac{\mathrm{d} V(t)}{\mathrm{d} t}+i_{\mathrm{C}}(t)
$$

where

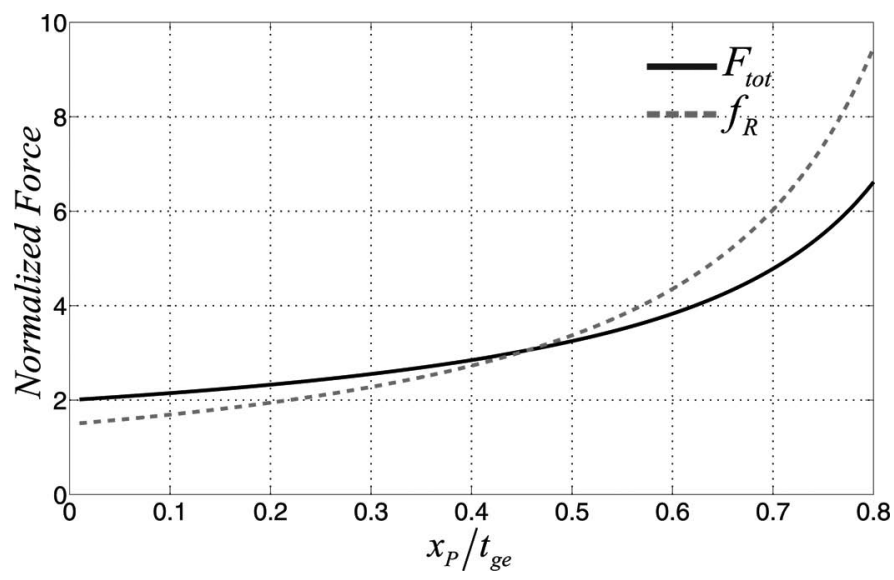

Fig. 2. A comparison of $F_{\text {tot }}$ and $f_{\mathrm{R}}$ normalized with $C_{0} V^{2}(t) / 4 t_{\mathrm{ge}}$ for a full electrode membrane.

$$
i_{\mathrm{C}}(t)=\left(C(t)-C_{0}\right) \frac{\mathrm{d} V(t)}{\mathrm{d} t}
$$

The velocity current is given by

$$
i_{\mathrm{V}}(t)=\frac{\partial C(t)}{\partial t} V(t)=\frac{\partial C(t)}{\partial x_{\mathrm{R}}} \frac{\partial x_{\mathrm{R}}}{\partial t} V(t) .
$$

Using (6), (7), and $C(t)=2 E(t) / V^{2}(t)$, we find

$$
i_{\mathrm{V}}(t)=\frac{2 f_{\mathrm{R}}(t)}{V(t)} v_{\mathrm{R}}(t)=\sqrt{5} \frac{C_{0} V(t)}{t_{\text {ge }}} g^{\prime}\left(\frac{x_{\mathrm{P}}(t)}{t_{\text {ge }}}\right) v_{\mathrm{R}}(t) .
$$

Eqs. (16) and (18) are the same as the corresponding equations in [1]. We can form the large signal equivalent circuit as depicted in Fig. 3. $C_{\mathrm{Rm}}$ and $L_{\mathrm{Rm}}$ are the compliance of the membrane and the inductance corresponding to the mass of the membrane suitable for the $\left\{f_{\mathrm{R}}, v_{\mathrm{R}}\right\} \mathrm{rms}$ model. For the same model, $Z_{\mathrm{RR}}$ is the radiation impedance of the CMUT cell given in [1].

Because the direction of $x_{\mathrm{P}}$ is chosen toward the bottom electrode and the particle velocity of the acoustic signal propagating into the medium is in the opposite direction, we denote the polarity of the transmitted force, $f_{\mathrm{RO}}$, across the radiation impedance, as shown in the figure. Similarly, any dynamic and static external force, such as an incident acoustic signal or atmospheric pressure, must appear in the form of $f_{\mathrm{RI}}$ and $F_{\mathrm{Rb}}$, respectively, in the model.

For the velocity profile given by (1), the average velocity, $v_{\mathrm{A}}(t)$, across the membrane is equal to $v_{\mathrm{A}}(t)=v_{\mathrm{P}}(t) / 3$. If $v_{\mathrm{A}}(t)$ is the through variable, the across variable is $f_{\mathrm{A}}(t)$ $=3 f_{\mathrm{R}}(t) / \sqrt{5}$, which preserves energy in the $\left\{f_{\mathrm{A}}, v_{\mathrm{A}}\right\}$ model. Similarly, if $v_{\mathrm{P}}(t)=\mathrm{d} x_{\mathrm{P}}(t) / \mathrm{d} t$ is used as the through variable, $f_{\mathrm{P}}(t)=f_{\mathrm{R}}(t) / \sqrt{5}$ is the force variable. In all cases, the mechanical circuit components must be scaled properly to be consistent and equivalent. The circuit components for all of these models are listed in Table I.

\section{Collapse}

To quantify the collapse phenomenon, we consider the circuit of Fig. 4 for the $\left\{f_{\mathrm{P}}, v_{\mathrm{P}}\right\}$ peak model to examine the static behavior under collapse conditions when an external static force $F_{\mathrm{Pb}}$ is present. We apply a voltage of $V_{\mathrm{DC}}$ to get the force $F_{\mathrm{P}}$ and the static displacement $X_{\mathrm{P}}$. The static force equilibrium in the mechanical section can be written as

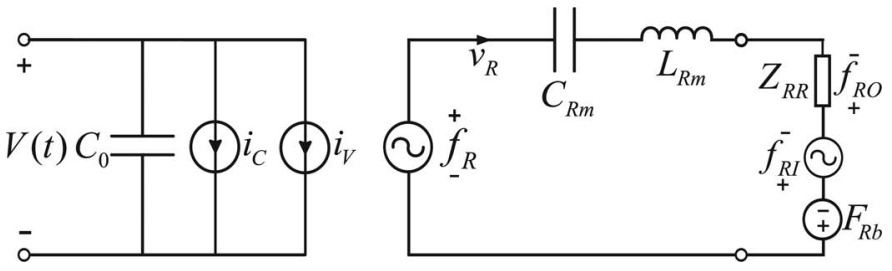

Fig. 3. Large signal equivalent circuit referred to as the $\left\{f_{\mathrm{R}}, v_{\mathrm{R}}\right\}$ model, because the through variable in the mechanical section is $v_{\mathrm{R}}$. 
Table I. Relations Between the Mechanical Variables of Different Models for the Equivalent Circuit Given in Fig. 4, and Turns Ratio and Spring Softening Compliance in the Small Signal Model.

\begin{tabular}{llll}
\hline Model & $\begin{array}{l}\text { RMS } \\
\left\{f_{\mathrm{R}}, v_{\mathrm{R}}\right\}\end{array}$ & $\begin{array}{l}\text { Average } \\
\left\{f_{\mathrm{A}}, v_{\mathrm{A}}\right\}\end{array}$ & $\begin{array}{l}\text { Peak } \\
\left\{f_{\mathrm{P}}, v_{\mathrm{P}}\right\}\end{array}$ \\
\hline$f$ & $f_{\mathrm{R}}$ & $(3 / \sqrt{5}) f_{\mathrm{R}}$ & $(1 / \sqrt{5}) f_{\mathrm{R}}$ \\
$v$ & $v_{\mathrm{R}}$ & $(\sqrt{5} / 3) v_{\mathrm{R}}$ & $\sqrt{5} v_{\mathrm{R}}$ \\
$C_{\mathrm{M}}$ & $C_{\mathrm{Rm}}=\frac{9}{5} \frac{\left(1-\sigma^{2}\right) a^{2}}{16 \pi Y_{0} t_{\mathrm{m}}^{3}}$ & $C_{\mathrm{Am}}=5 / 9 C_{\mathrm{Rm}}$ & $C_{\mathrm{Pm}}=5 C_{\mathrm{Rm}}$ \\
$L_{\mathrm{M}}$ & $L_{\mathrm{Rm}}=\rho \pi a^{2} t_{\mathrm{m}}$ & $L_{\mathrm{Am}}=9 / 5 L_{\mathrm{Rm}}$ & \\
$Z_{\mathrm{R}}$ & $Z_{\mathrm{RR}}$ & $Z_{\mathrm{AR}}=9 / 5 Z_{\mathrm{RR}}$ & $L_{\mathrm{Pm}}=1 / 5 L_{\mathrm{Rm}}$ \\
$f_{\mathrm{I}}$ & $\pi a^{2} p_{\text {in }}$ & $(3 / \sqrt{5}) \pi a^{2} p_{\text {in }}$ & $Z_{\mathrm{PR}}=1 / 5 Z_{\mathrm{RR}}$ \\
$f_{\mathrm{O}}$ & $\pi a^{2} p_{\mathrm{o}}$ & $(3 / \sqrt{5}) \pi a^{2} p_{\mathrm{o}}$ & $(1 / \sqrt{5}) \pi a^{2} p_{\mathrm{in}}$ \\
$F_{\mathrm{b}}$ & $(\sqrt{5} / 3) \pi a^{2} P_{0}$ & $\pi a^{2} P_{0}$ & $(1 / \sqrt{5}) \pi a^{2} p_{\mathrm{o}}$ \\
$n$ & $n_{\mathrm{R}}$ & $n_{\mathrm{A}}=(3 / \sqrt{5}) n_{\mathrm{R}}$ & $1 / 3 \pi a^{2} P_{0}$ \\
$C_{\mathrm{S}}$ & $C_{\mathrm{RS}}$ & $C_{\mathrm{AS}}=5 / 9 C_{\mathrm{RS}}$ & $n_{\mathrm{P}}=(1 / \sqrt{5}) n_{\mathrm{R}}$ \\
\hline
\end{tabular}

$p_{\text {in }}$ and $p_{\mathrm{o}}$ are the incident and transmitted wave pressures at the radiation interface, respectively.

$$
F_{\mathrm{P}}+F_{\mathrm{Pb}}=\frac{X_{\mathrm{P}}}{C_{\mathrm{Pm}}}
$$

which yields

$$
\frac{V_{\mathrm{DC}}}{V_{\mathrm{r}}}=\sqrt{\frac{3\left(\frac{X_{\mathrm{P}}}{t_{\mathrm{ge}}}-\frac{F_{\mathrm{Pb}}}{F_{\mathrm{Pg}}}\right)}{2 g^{\prime}\left(\frac{X_{\mathrm{P}}}{t_{\mathrm{ge}}}\right)}} \text { for } \frac{X_{\mathrm{P}}}{t_{\mathrm{ge}}} \geq \frac{F_{\mathrm{Pb}}}{F_{\mathrm{Pg}}},
$$

where we define $V_{\mathrm{r}}$ as

$$
V_{\mathrm{r}}=\sqrt{\frac{4 t_{\mathrm{ge}}^{2}}{3 C_{\mathrm{Pm}} C_{0}}}=8 \frac{t_{\mathrm{m}}}{a^{2}} t_{\mathrm{ge}}^{3 / 2} t_{\mathrm{m}}^{1 / 2} \sqrt{\frac{Y_{0}}{27 \varepsilon_{0}\left(1-\sigma^{2}\right)}}
$$

and $F_{\mathrm{Pg}}=t_{\mathrm{ge}} / C_{\mathrm{Pm}}$ is the force required to deflect the membrane until the center displacement reaches the gap height, $x_{\mathrm{P}}=t_{\mathrm{ge}} . V_{\mathrm{DC}} / V_{\mathrm{r}}$ for a CMUT with full electrodes is plotted in Fig. 5 with respect to $X_{\mathrm{P}} / t_{\mathrm{ge}}$ for $F_{\mathrm{Pb}} / F_{\mathrm{Pg}}=$ $0,0.1,0.5,0.7$, and 0.9 .

It can be observed from Fig. 5 that the bias voltage can be increased until it reaches a maximum for a particular external static force and the equilibrium is stable in this region. If the voltage is increased beyond the maximum, the transduction force exceeds the restoring force and collapse occurs. Bias voltage must be decreased to maintain equilibrium in this region. This equilibrium is unstable.

The figure reveals the relation of collapse phenomena, the bias voltage, the static force, and $V_{\mathrm{r}}$. For example, there is no static force in vacuum and the bias voltage

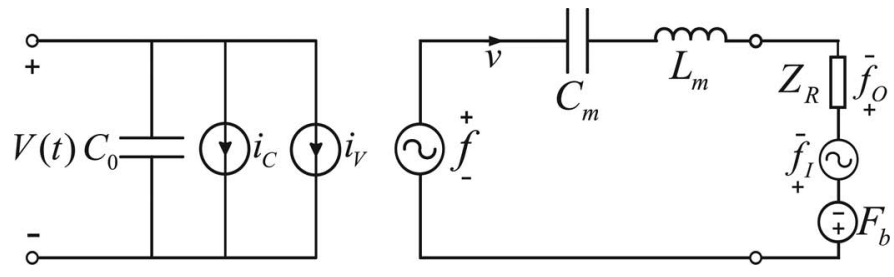

Fig. 4. Generic large signal equivalent circuit model with parameters given in Table I. maximum is $1.000476 V_{\mathrm{r}}$, hence the collapse voltage of a CMUT in vacuum can be taken as $V_{\mathrm{r}}$. In the presence of a static force, such as atmospheric pressure, membrane is pre-depressed by this force and collapse occurs at a bias voltage less than $V_{\mathrm{r}}$.

It is clear from Fig. 5 and (20) that the displacement threshold for collapse for any $F_{\mathrm{Pb}} / F_{\mathrm{Pg}}$ is reached when $V_{\mathrm{DC}} / V_{\mathrm{r}}$ is maximum. Hence, the displacement at collapse point, $X_{\mathrm{Pc}}$, is obtained from

$$
\left.\frac{\mathrm{d}}{\mathrm{d} X_{\mathrm{P}}}\left(\frac{V_{\mathrm{DC}}}{V_{\mathrm{r}}}\right)\right|_{X_{\mathrm{P}}=X_{\mathrm{Pc}}}=0
$$

while the equilibrium condition in (20) is maintained. $X_{\mathrm{Pc}}$ can be readily evaluated from (20). For membranes with full electrodes, a very accurate approximation is

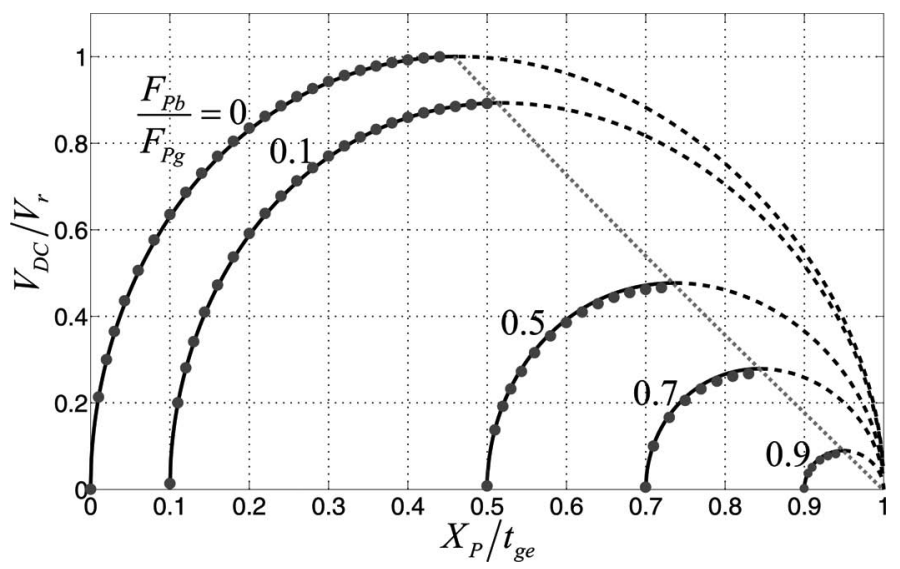

Fig. 5. The voltage at the stable (solid) and unstable (dashed) static equilibrium as a function of $F_{\mathrm{Pb}} / F_{\mathrm{Pg}}$ for different $X_{\mathrm{P}}$ values for a membrane with full electrodes with the properties given in Section IV. The straight line shows the variation of the voltage required to reach the collapse point for all $F_{\mathrm{Pb}} / F_{\mathrm{Pg}}$. In the static FEM analysis results (dotted), the stress stiffening effects are ignored. 


$$
\begin{aligned}
\frac{X_{\mathrm{Pc}}}{t_{\mathrm{ge}}} \approx & 0.4648+0.5433 \frac{F_{\mathrm{Pb}}}{F_{\mathrm{Pg}}}-0.01256\left(\frac{F_{\mathrm{Pb}}}{F_{\mathrm{Pg}}}-0.35\right)^{2} \\
& -0.002775\left(\frac{F_{\mathrm{Pb}}}{F_{\mathrm{Pg}}}\right)^{9} .
\end{aligned}
$$

The voltage, $V_{\mathrm{c}}$, required to reach $X_{\mathrm{Pc}}$ can be obtained by using (23) in (20). The variation of $V_{\mathrm{c}}$ with respect to $F_{\mathrm{Pb}} / F_{\mathrm{Pg}}$ is essentially a straight line and can be approximated as

$$
\begin{aligned}
\frac{V_{\mathrm{c}}}{V_{\mathrm{r}}} \approx & 0.9961-1.0468 \frac{F_{\mathrm{Pb}}}{F_{\mathrm{Pg}}}+0.06972\left(\frac{F_{\mathrm{Pb}}}{F_{\mathrm{Pg}}}-0.25\right)^{2} \\
& +0.01148\left(\frac{F_{\mathrm{Pb}}}{F_{\mathrm{Pg}}}\right)^{6} .
\end{aligned}
$$

Eq. (24) versus (23) is also plotted in Fig. 5 as the collapse threshold. Similarly, the $F_{\mathrm{Pb}} / F_{\mathrm{Pg}}$ ratio can also be approximated very accurately in terms of $V_{\mathrm{c}} / V_{\mathrm{r}}$ as

$$
\begin{aligned}
\frac{F_{\mathrm{Pb}}}{F_{\mathrm{Pg}}} \approx & 0.9891-1.037 \frac{V_{\mathrm{c}}}{V_{\mathrm{r}}}+0.2083\left(\frac{V_{\mathrm{c}}}{V_{\mathrm{r}}}-0.229\right)^{2} \\
& -0.0755\left(\frac{V_{\mathrm{c}}}{V_{\mathrm{r}}}\right)^{3} .
\end{aligned}
$$

\section{Received and Transmitted Pressure}

$f_{\mathrm{I}}$ and $f_{\mathrm{O}}$ are received and transmitted forces of the mod$\mathrm{el}$, respectively. It is more convenient if these are expressed in terms of the pressure at the surface of the membrane. When an equivalent model is produced, transducers of any kind are converted into a rigid piston transducer with uniformly distributed velocity and displacement, $v$ and $x$, respectively, across its radiating surface. All power and energy conversion at the radiating interface is expressed by these lumped variables.

CMUTs cannot produce a static output pressure in infinite fluid volume. There is no radiation impedance for static signals. When a static pressure $P_{0}$ is present in the medium, the total force on the membrane is $\pi a^{2} P_{0}$. The work done on a narrow ring by this static pressure can be obtained as

$$
\delta E=\left(P_{0} 2 \pi r \mathrm{~d} r\right) \int_{0}^{x(r)} \mathrm{d} x=\left(P_{0} 2 \pi r \mathrm{~d} r\right) x(r) .
$$

Then, the total work is found as

$$
E=P_{0} 2 \pi X_{\mathrm{P}} \int_{0}^{a}\left(1-\frac{r^{2}}{a^{2}}\right)^{2} r \mathrm{~d} r=\frac{\pi a^{2} P_{0}}{3} X_{\mathrm{P}} .
$$

It is clear from (27) that $\pi a^{2} P_{0}$ corresponds to the input static force in the average model $\left\{f_{\mathrm{A}}, v_{\mathrm{A}}\right\}$.
For dynamic signals, we consider the power relation at the radiation interface. The acoustic power intercepted by a receiving transducer from an incident plane wave can be expressed in terms of the particle velocity in the medium, $v_{\mathrm{m}}$, as $\pi a^{2} \rho c\left(v_{\mathrm{m}} v_{\mathrm{m}}^{*}\right)$. The power emitted to the medium from a rigid piston with a large aperture compared with the wavelength, can be expressed similarly. The same power written in terms of velocity distribution on the membrane yields:

$$
\rho c \int_{0}^{a} v_{\mathrm{P}} v_{\mathrm{P}}^{*}\left(1-\frac{r^{2}}{a^{2}}\right)^{4} 2 \pi r \mathrm{~d} r=\pi a^{2} \rho c\left(v_{\mathrm{R}} v_{\mathrm{R}}^{*}\right)
$$

Therefore, when the radiated power is expressed in terms of the through variable only, the rms velocity maintains the consistency between transducer output and radiated power. We conclude that the forces obtained by multiplying the equivalent uniform dynamic pressures on the membrane surface by membrane area are the lumped forces at the output terminals of the rms equivalent circuit. The forces obtained from the dynamic field pressures must be scaled when used in other two models. These relations are given in Table I.

The power delivered to the medium is the same in all three models. However, the force, and hence the equivalent uniform pressure delivered to the medium, is scaled in the $\left\{f_{\mathrm{P}}, v_{\mathrm{P}}\right\}$ and $\left\{f_{\mathrm{A}}, v_{\mathrm{A}}\right\}$ models according to the associated through variable and is different than the product of the area and the pressure in the field. The only through variable which produces an equivalent circuit whose dynamic output pressure is compatible with the field pressure is $v_{\mathrm{R}}$. Consequently, there is no need to scale the received dynamic pressure in the rms model. For example, when the output velocity is used in beam-forming, the particle velocity at the output of the rms equivalent circuit corresponds to the physical particle velocity of the CMUT cell. In [23], $v_{\mathrm{R}}$ is shown to be the suitable reference lumped velocity for diffraction constant calculations in transducers.

\section{E. Spring Constant of the Membrane}

The accuracy of the equivalent circuit presented in this paper depends on two factors: the agreement of the actual velocity profile with the assumed one and the accuracy of mechanical circuit elements and the radiation impedance. A CMUT cell with a circular membrane, $a / t_{\mathrm{m}} \geq 80$, is assumed and the compliance of the membrane, $C_{\mathrm{m}}$, is taken as in Table I. It can be shown by FEM analysis that although both profile and expression in Table I are excellent models at very low center displacement, they deviate from these as center displacement increases. Particularly, $C_{\mathrm{m}}$ decreases significantly because the membrane gets stiffer [24], [25].

The membranes of CMUTs often fall into the plate class $\left(a / t_{\mathrm{m}}<80\right)[24]$. As the plate gets thicker, its compliance becomes softer than the value calculated from Table I. 
The lumped element models require only a lump-sum, but correct, assessment of the effect. Both the collapse voltage and the resonance dynamics depend on the membrane compliance. If the dependence of this compliance to the physical dimensions of the membrane is adequately modeled and included into the equivalent circuit, the accuracy of the model predictions for thicker plates increases. A comprehensive model for $C_{\mathrm{m}}$ nonlinearity that is applicable for all possible $a / t_{\mathrm{m}}$ ratios and material properties is not addressed in this work. Nevertheless, it is shown in Section IV that even without any correction for profile or $C_{\mathrm{m}}$, the equivalent circuit produces very accurate results.

\section{Small Signal Model}

Almost all reception operations are small signal applications. A small signal equivalent circuit can be derived from the large signal model. We consider the $\left\{f_{\mathrm{R}}, v_{\mathrm{R}}\right\}$ model and make the small signal assumptions: we assume that the ac voltage at the device terminal is small and write

$$
V^{2}(t)=\left[V_{\mathrm{DC}}+V_{\mathrm{ac}}(t)\right]^{2} \approx V_{\mathrm{DC}}^{2}+2 V_{\mathrm{DC}} V_{\mathrm{ac}}(t)
$$

because $\left|V_{\mathrm{ac}}(t)\right| \ll V_{\mathrm{DC}}$. We write the displacement $\mathrm{as}^{2}$

$$
x_{\mathrm{R}}(t)=X_{\mathrm{R}}+x_{\mathrm{r}}(t) \quad \text { with }\left|x_{\mathrm{r}}(t)\right| \ll X_{\mathrm{R}} .
$$

$f_{\mathrm{R}}(t)$ given by $(7)$ in the large signal model can be linearized around $X_{\mathrm{R}}$ as

$$
f_{\mathrm{R}}(t)=F_{\mathrm{R}}+f_{\mathrm{r}}(t)=\left.f_{\mathrm{R}}\right|_{x_{\mathrm{R}}=X_{\mathrm{R}}}+\left.\frac{\mathrm{d} f_{\mathrm{R}}}{\mathrm{d} x_{\mathrm{R}}}\right|_{x_{\mathrm{R}}=X_{\mathrm{R}}} x_{\mathrm{r}}(t) .
$$

Ignoring the second-order terms, the force terms become

$$
\begin{gathered}
F_{\mathrm{R}}=\sqrt{5} \frac{C_{0} V_{\mathrm{DC}}^{2}}{2 t_{\mathrm{ge}}} g^{\prime}\left(\frac{X_{\mathrm{P}}}{t_{\mathrm{ge}}}\right) \\
f_{\mathrm{r}}(t) \approx \frac{2 F_{\mathrm{R}}}{V_{\mathrm{DC}}} V_{\mathrm{ac}}(t)+\sqrt{5} \frac{C_{0} V_{\mathrm{DC}}^{2}}{2 t_{\mathrm{ge}}^{2}} g^{\prime \prime}\left(\frac{X_{\mathrm{P}}}{t_{\mathrm{ge}}}\right) x_{\mathrm{p}}(t) .
\end{gathered}
$$

Eq. (32) is the dc force which provides the static deflection. $g^{\prime \prime}(\cdot)$ is given by (9). From (33), we obtain the linear transduction equation in rms variables as

$$
f_{\mathrm{r}}(t)=n_{\mathrm{R}} V_{\mathrm{ac}}(t)+\frac{x_{\mathrm{r}}(t)}{C_{\mathrm{RS}}},
$$

where

$$
n_{\mathrm{R}}=\frac{2 F_{\mathrm{R}}}{V_{\mathrm{DC}}}
$$

\footnotetext{
${ }^{2}$ Capital letters with capital subscripts refer to dc quantities, whereas lowercase letters with lowercase subscripts are small signal quantities.
}

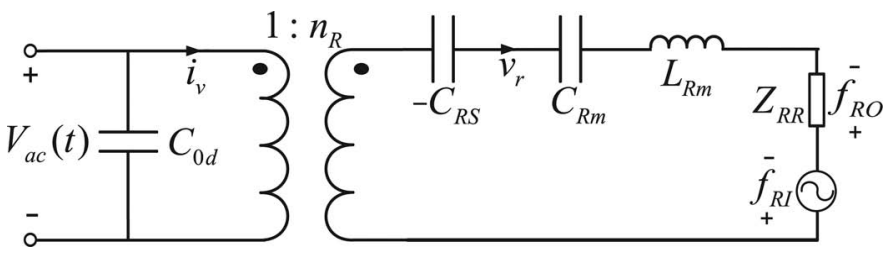

Fig. 6. Small signal equivalent circuit for the $\left\{f_{\mathrm{R}}, v_{\mathrm{R}}\right\}$ model.

is the electromechanical turns ratio at the operating point and

$$
C_{\mathrm{RS}}=\frac{2 t_{\mathrm{ge}}^{2}}{5 C_{0} V_{\mathrm{DC}}^{2} g^{\prime \prime}\left(X_{\mathrm{P}} / t_{\mathrm{ge}}\right)}
$$

is the spring softening capacitor. We note that a linearization of (18) around the operating point gives

$$
i_{\mathrm{v}}=\frac{2 F_{\mathrm{R}}}{V_{\mathrm{DC}}} v_{\mathrm{r}}(t)=n_{\mathrm{R}} v_{\mathrm{r}}(t)
$$

consistent with the turns ratio definition of (35).

The only small signal component on the electrical side is the capacitance of the deflected membrane found when (3) is linearized at the operating point:

$$
C_{0 \mathrm{~d}}=C_{0} g\left(\frac{X_{\mathrm{P}}}{t_{\mathrm{ge}}}\right) .
$$

The small signal equivalent circuit with these components is depicted in Fig. 6.

To evaluate circuit parameters $C_{0 \mathrm{~d}}, n_{\mathrm{R}}$, and $C_{\mathrm{RS}}$, we first specify $X_{\mathrm{R}} / t_{\mathrm{ge}}$ such that $X_{\mathrm{R}} / t_{\mathrm{ge}}<X_{\mathrm{Pc}} /\left(\sqrt{5} t_{\mathrm{ge}}\right)$ for the operating $V_{\mathrm{DC}}$ and the static force $F_{\mathrm{b}}$ using $(23)$ and then evaluate the circuit parameters using (35), (36), and (38).

We follow the procedures given in Section II-B to get the equivalent circuits for other through variables. The turns ratio and the spring softening compliance for peak and average equivalent circuits are given in Table I. $C_{0 \mathrm{~d}}$ remains unchanged.

\section{Comparison With FEM Analysis}

The predictions of the equivalent circuit model are examined through FEM analyses. Static, prestressed harmonic, and nonlinear transient analyses are performed using the simulation package Ansys v13 (Ansys Inc., Canonsburg, PA). In all simulations, an immersed CMUT cell with a silicon nitride membrane is used, whose material properties are taken as $\rho=3.27 \mathrm{~g} / \mathrm{cm}^{3}, Y_{0}=320 \mathrm{GPa}$ and $\sigma=0.263$. The density and the speed of sound in water are taken as $1 \mathrm{~g} / \mathrm{cm}^{3}$ and $1500 \mathrm{~m} / \mathrm{s}$, respectively.

In Fig. 7, a comparison is made between the prediction of the equivalent model and the FEM model, based on the conductance of a CMUT cell in water. In FEM simulations, an absorbing boundary layer is employed, which simulates a fluid domain that extends to infinity beyond 


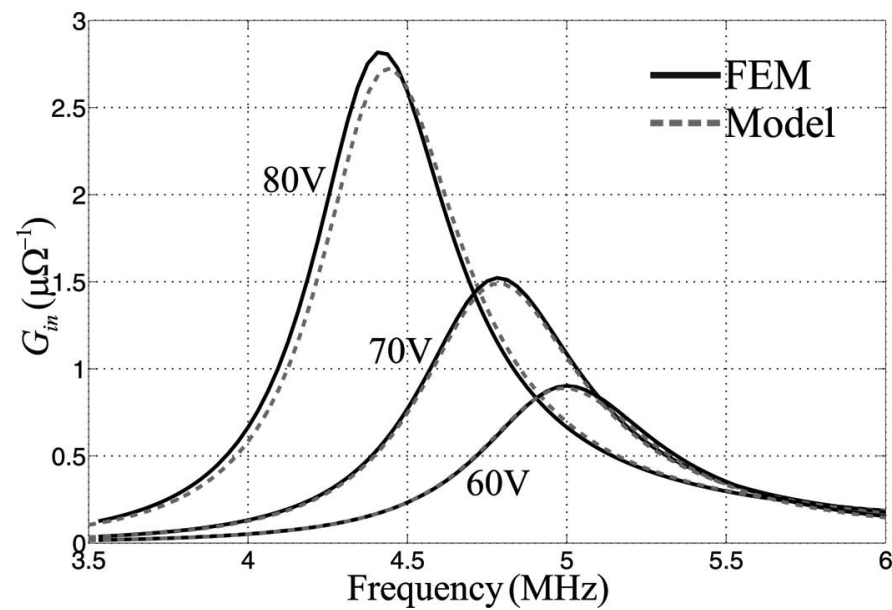

Fig. 7. Small signal conductance of a silicon nitride $\left(\mathrm{Si}_{3} \mathrm{Ni}_{4}\right)$ membrane capacitive micromachined ultrasonic transducer (CMUT) in water with $a=20 \mu \mathrm{m}, t_{\mathrm{ge}}=250 \mathrm{~nm}, t_{\mathrm{m}}=1 \mu \mathrm{m}$. A $1-\mathrm{V}$ ac signal is applied with 60, 70, and $80 \mathrm{~V}$ bias voltages. Finite element method (FEM; solid line) results are acquired from prestressed harmonic analyses and compared with the frequency response of the model (dashed line).

the boundary. Although it is preferable to use a 2-D axisymmetric FEM model for a single CMUT cell, we used a 3-D FEM model for all prestressed harmonic analyses. We realized that in 2-D FEM models, the resonance frequency and the amplitude of the harmonic response change depending on the distance between the absorbing boundary layer and the CMUT. However, we did not observe this problem in 3-D FEM models when the absorbing boundary layer is located at least $0.2 \lambda+a$ away from the center of the CMUT cell, as suggested by Ansys. Here, $\lambda$ is taken as the greatest wavelength of the pressure waves for that analysis.

The membrane of this CMUT is quite thick $\left(a / t_{\mathrm{m}}=\right.$ 20). The model employs the thin plate compliance for membrane and this contributes to the difference in the

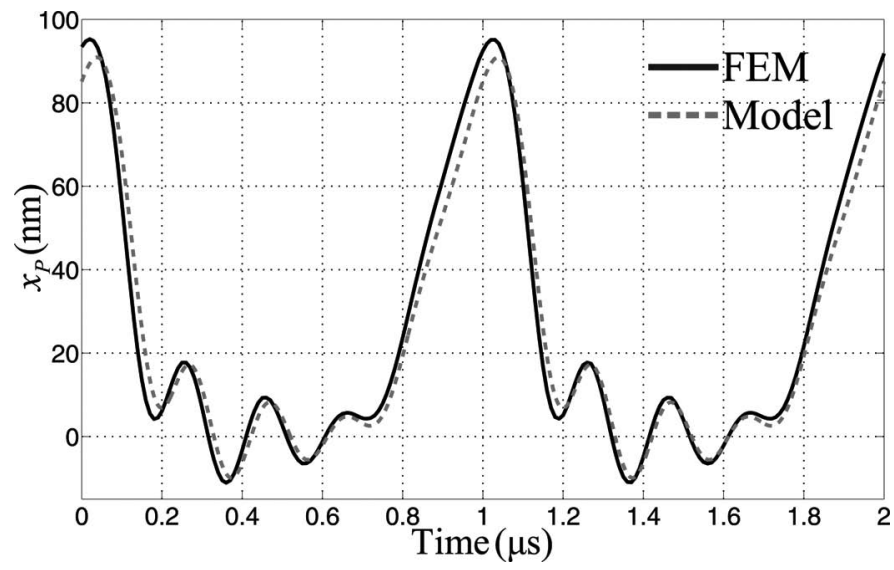

Fig. 8. Peak displacement of the capacitive micromachined ultrasonic transducer (CMUT) cell in water with $a=20 \mu \mathrm{m}, t_{\mathrm{ge}}=250 \mathrm{~nm}, t_{\mathrm{m}}=$ $1 \mu \mathrm{m}$, which is driven with $50 \mathrm{~V}$ peak ac voltage and $40 \mathrm{~V}$ bias voltage. The frequency of the applied signal is one-fifth the resonance frequency of the transducer. Steady-state time domain response of the model (dashed line) is compared with the one obtained with the finite element method (FEM; solid line).

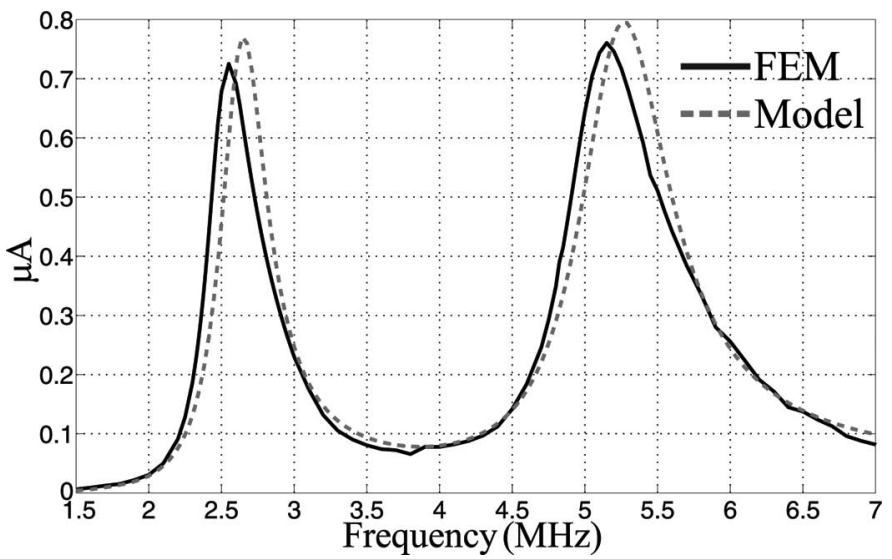

Fig. 9. Real part of the fundamental source current flowing through a silicon nitride $\left(\mathrm{Si}_{3} \mathrm{Ni}_{4}\right)$ membrane capacitive micromachined ultrasonic transducer (CMUT) cell in water with $a=20 \mu \mathrm{m}, t_{\mathrm{ge}}=250 \mathrm{~nm}, t_{\mathrm{m}}=$ $1 \mu \mathrm{m}$. A $40 \mathrm{~V}$ peak ac voltage is applied on $10 \mathrm{~V}$ bias voltage. Large signal response is observed in finite element method (FEM, solid line) transient analysis and compared with the response of the model shown in Fig. 3 (dashed line).

resonance frequencies predicted by the model and by FEM analysis.

The large signal performance of the model is compared with the FEM results on the same CMUT cell, but under extreme electrical drive conditions, which emphasize the nonlinear effects. In Fig. 8, the model and FEM predictions are depicted for a CMUT biased with $40 \mathrm{~V}$ and driven with a sinusoidal signal of $50 \mathrm{~V}$ peak amplitude at $1 \mathrm{MHz}$. For reference, the small signal resonance frequency under $40 \mathrm{~V}$ dc bias is $5.3 \mathrm{MHz}$. Time domain steady state response of the model is compared with the transient analysis in FEM. The nonlinearity is very noticeable, because the amplitude of the ac signal is large and the frequency is approximately one-fifth of the resonance frequency of this CMUT. FEM and model predictions are very consistent.

The large signal performance of the model is further studied and a peak is observed in the real part of the fundamental component of the source current at half the resonance frequency. This can be explained as follows: the generated force is proportional to the square of the applied voltage and the second harmonic is inherently present in the generated force. The second-harmonic component increases very significantly at high sinusoidal drive levels. When the second-harmonic frequency of the applied voltage coincides with the resonance frequency, there is an efficient acoustic radiation and the current drawn from the source increases. We repeated this analysis in which $40 \mathrm{~V}$ peak sinusoidal voltage and $10 \mathrm{~V}$ bias voltage are applied to the same CMUT cell in water, which has a collapse voltage of $95 \mathrm{~V}$. As shown in Fig. 9, the FEM and lumped element model results agree very well.

\section{CONCLUSION}

We presented a lumped element equivalent circuit that can predict the entire behavior of a circular CMUT cell 
operated in the uncollapsed mode. Rigid membrane supports and a rigid substrate are assumed in deriving the model, hence it does not include the loss to the substrate and cross-talk through the substrate. We found that a correct evaluation of membrane compliance is critical for the accuracy of model predictions and in determining the collapse voltage.

\section{REFERENCES}

[1] H. K. Oguz, S. Olcum, M. N. Senlik, V. Tas, A. Atalar, and H. Köymen, "Nonlinear modeling of an immersed transmitting capacitive micromachined ultrasonic transducer for harmonic balance analysis," IEEE Trans. Ultrason. Ferroelectr. Freq. Control, vol. 57, no. 2, pp. $438-447,2010$.

[2] I. O. Wygant, M. Kupnik, and B. T. Khuri-Yakub, "Analytically calculating membrane displacement and the equivalent circuit model of a circular CMUT cell," in Proc. IEEE Ultrasonics Symp., 2008, pp. 2111-2114.

[3] S. Olcum, F. Yamaner, A. Bozkurt, H. Köymen, and A. Atalar, "An equivalent circuit model for transmitting capacitive micromachined ultrasonic transducers in collapse mode," IEEE Trans. Ultrason. Ferroelectr. Freq. Control, vol. 58, no. 7, pp. 1468-1477, 2011.

[4] A. Lohfink and P. C. Eccardt, "Linear and nonlinear equivalent circuit modeling of CMUTs," IEEE Trans. Ultrason. Ferroelectr. Freq. Control, vol. 52, no. 12, pp. 2163-2172, 2005.

[5] C. Doody, X. Cheng, C. Rich, D. Lemmerhirt, and R. White, "Modeling and characterization of CMOS-fabricated capacitive micromachined ultrasound transducers," J. Microelectromech. Syst., vol. 20, no. 1, pp. 104-118, 2011.

[6] A. Caronti, G. Caliano, A. Iula, and M. Pappalardo, "An accurate model for capacitive micromachined ultrasonic transducers," IEEE Trans. Ultrason. Ferroelectr. Freq. Control, vol. 49, no. 2, pp. 159-168, 2002.

[7] D. Certon, F. Teston, and F. Patat, "A finite difference model for CMUT devices," IEEE Trans. Ultrason. Ferroelectr. Freq. Control, vol. 52, no. 12, pp. 2199-2210, Dec. 2005.

[8] G. G. Yaralioglu, A. S. Ergun, and B. T. Khuri-Yakub, "Finite-element analysis of capacitive micromachined ultrasonic transducers," IEEE Trans. Ultrason. Ferroelectr. Freq. Control, vol. 52, no. 12, pp. 2185-2198, 2005.

[9] A. Bozkurt, "Finite element modeling of CMUTs using a perfectly matched layer for fast simulation," in Proc. IEEE Ultrasonics Symp., 2003, pp. 1979-1982.

[10] B. Bayram, G. G. Yaralioglu, A. S. Ergun, Ö. Oralkan, and B. T. Khuri-Yakub, "Dynamic FEM analysis of multiple CMUT cells in immersion," in Proc. IEEE Ultrasonics Symp., 2004, pp. 252-255.

[11] D.-S. Lin, X. Zhuang, S. Wong, M. Kupnik, and B. Khuri-Yakub, "Encapsulation of capacitive micromachined ultrasonic transducers using viscoelastic polymer," J. Microelectromech. Syst., vol. 19, no. 6, pp. 1341-1351, 2010.

[12] B. Bayram, M. Kupnik, G. Yaralioglu, O. Oralkan, A. Ergun, S. Wong, and B. Khuri-Yakub, "Finite element modeling and experimental characterization of crosstalk in 1-D CMUT arrays," IEEE Trans. Ultrason. Ferroelectr. Freq. Control, vol. 54, no. 2, pp. 418430, 2007.

[13] F. L. Degertekin, R. O. Guldiken, and M. Karaman, "Annular-ring CMUT arrays for forward-looking IVUS: Transducer characterization and imaging," IEEE Trans. Ultrason. Ferroelectr. Freq. Control, vol. 53 , no. 2 , pp. $474-482,2006$.

[14] X. Jin, I. Ladabaum, and B. T. Khuri-Yakub, "The microfabrication of capacitive micromachined ultrasonic transducers," J. Microelectromech. Syst., vol. 7, no. 3, pp. 295-302, 1998.

[15] Y. Huang, A. S. Ergun, E. Hæggström, M. H. Badi, and B. T. Khuri-Yakub, "Fabricating capacitive micromachined ultrasonic transducers with wafer-bonding technology," J. Microelectromech. Syst., vol. 12, no. 2, pp. 128-137, 2003.

[16] M. N. Senlik, S. Olcum, H. Köymen, and A. Atalar, "Radiation impedance of an array of circular capacitive micromachined ultrasonic transducers," IEEE Trans. Ultrason. Ferroelectr. Freq. Control, vol. 57, no. 4, pp. 969-976, 2010.
[17] K. Park, M. Kupnik, H. Lee, B. Khuri-Yakub, and I. Wygant, "Modeling and measuring the effects of mutual impedance on multicell CMUT configurations," in Proc. IEEE Ultrasonics. Symp., 2010, pp. 431-434.

[18] A. Ozgurluk, A. Atalar, H. Koymen, and S. Olcum, "Radiation impedance of an array of circular capacitive micromachined ultrasonic transducers in collapsed state," in Proc. IEEE Ultrasonics Symp., 2011.

[19] W. P. Mason, Electromechanical Transducers and Wave Filters. New York, NY: Van Nostrand, 1942.

[20] M. Greenspan, "Piston radiator: Some extensions of the theory," $J$. Acoust. Soc. Am., vol. 65, no. 3, pp. 608-621, 1979.

[21] S. Timoshenko and S. Woinowsky-Krieger, Theory of Plates and Shells, 2nd ed., New York, NY: McGraw Hill, 1959.

[22] C. E. Wallace, "Radiation resistance of a baffled beam," J. Acoust. Soc. Am., vol. 51, no. 3B, pp. 936-945, 1972.

[23] R. S. Woollett, "Diffraction constants for pressure gradient transducers," J. Acoust. Soc. Am., vol. 72, no. 4, pp. 1105-1113, 1982.

[24] E. Ventsel and T. Krauthammer, Thin Plates and Shells. New York, NY: Marcel Decker, 2001.

[25] M. Kupnik, I. Wygant, and B. Khuri-Yakub, "Finite element analysis of stress stiffening effects in CMUTs," in Proc. IEEE Ultrasonics Symp., 2008, pp. 487-490.

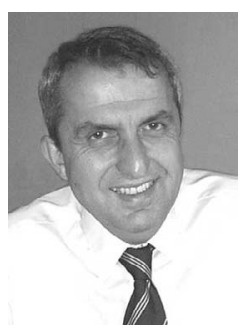

Hayrettin Köymen received the B.Sc. and M. Sc. degrees from the Middle East Technical University (METU), Ankara, Turkey, in 1973 and 1976, respectively, and the Ph.D. degree from Birmingham University, UK, in 1979, all in electrical engineering. He worked as a faculty member in the Marine Sciences Department (Mersin) and the Electrical Engineering Department (Ankara) of METU, from 1979 to 1990, and at Bilkent University since 1990, where he is a professor. His research activities have included underwater acoustic and ultrasonic transducer design, acoustic microscopy, ultrasonic NDT, biomedical instrumentation, mobile communications, and spectrum management.

Prof. Köymen is a fellow of IET (formerly IEE).

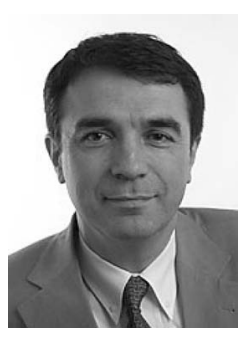

Abdullah Atalar received the B.S. degree from the Middle East Technical University, Ankara, Turkey, in 1974, and M.S. and Ph.D. degrees from Stanford University, Stanford, CA, in 1976 and 1978, respectively, all in electrical engineering. He worked in Hewlett Packard Labs, Palo Alto, in 1979. From 1980 to 1986 , he was on the faculty of the Middle East Technical University as an Assistant Professor. In 1986, he joined Bilkent University as the chairman of the Electrical and Electronics Engineering Department and served in the founding of the Department, where he is currently a Professor. In 1995 he was a visiting professor at Stanford University. From 1996 to 2010, he was the Provost of Bilkent University. He is presently the Rector of the same university. His current research interests include micromachined devices and microwave electronics.

Prof. Atalar was awarded the Science Award of TUBITAK in 1994. He is a Fellow of IEEE and a member of the Turkish Academy of Sciences.

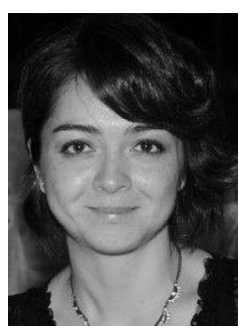

Elif Aydoğdu was born in Ankara, Turkey, in 1982. She received her B.S. and M.S. degrees in electronics engineering in 2004 and 2007, respectively, from Bilkent University, Ankara, Turkey. She is currently working toward her Ph.D. degree in the same department, where she has been a research assistant since 2004. Her current research interests include modeling and design of cMUTs. 


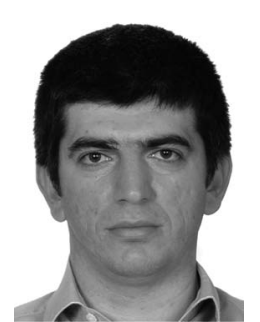

Coşkun Kocabaş received the B.Sc. degree from the Middle East Technical University, Ankara, Turkey, in 2001; the M.S. degree from Bilkent University, Ankara, Turkey, in 2003; and the Ph.D. degree from the University of Illinois at UrbanaChampaign, Urbana, IL, in 2007. Between 2007 and 2009, he worked at Harvard University, in the Department of Chemistry and Chemical Biology as a research fellow. He joined Bilkent University in 2009 as an Assistant Professor with appointments in the Department of Physics. His research interests include quantum electronics, nano-electromechanical systems, and lab-on-a-chip devices.

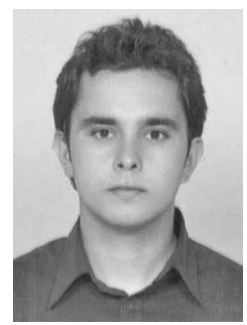

H. Kağan Oğuz was born in Ankara, Turkey, in 1985. He received his B.S. and M.S. degrees in electrical engineering in 2006 and 2009, respectively, both from Bilkent University, Ankara, Turkey. Between 2009 and 2012, he worked as an R\&D engineer at Meteksan Defence Industry Inc., Underwater Acoustic Systems Division, Ankara. Since 2009, he has been working toward his Ph.D. degree in the Electrical and Electronics Engineering Department at Bilkent University, where he is currently a research assistant. His current research interests include design and fabrication of underwater transducers and CMUTs.

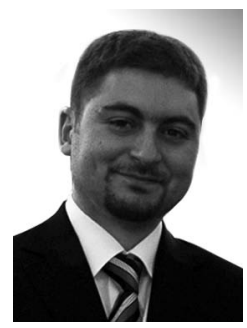

Selim Olçum was born in Chicago, IL, in 1981. He received his B.S., M.S., and Ph.D. degrees in electrical engineering in 2003, 2005, and 2010, respectively, all from Bilkent University, Ankara, Turkey. He worked as a guest researcher at the National Institute of Standards and Technology, Semiconductor Electronics Division, Gaithersburg, MD, during the summers of 2002 and 2003. He was a visiting scholar in the Micromachined Sensors and Transducers Laboratory of the Georgia Institute of Technology, Atlanta, GA, in 2006.
He was an instructor in the Electrical and Electronics Engineering Department at Bilkent University for six months in 2011. He is currently a postdoctoral associate in the Department of Biological Engineering and the Koch Institute for Integrative Cancer Research at the Massachusetts Institute of Technology (MIT), Cambridge, MA. His dissertation work was focused on developing high-performance micromachined ultrasonic transducers. His current research focus at MIT is to develop real-time techniques for biomolecular detection using micro- and nano-electromechanical devices.

Dr. Selim Olçum was a fellow of ASELSAN during his Ph.D. study.

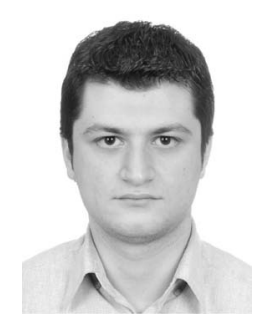

Alper Ozgurluk was born in Kirikkale, Turkey, in 1990. He is currently a senior undergraduate student in the Electrical and Electronics Engineering Department at Bilkent University, Ankara, Turkey.

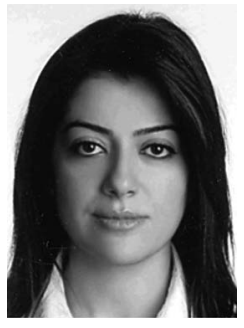

Asli Ünlügedik received a B.Sc. degree from the Electrical and Electronics Engineering Department, from Yeditepe University, Istanbul, Turkey, in 2004 and an M.Sc. degree from Istanbul Technical University (ITU), Istanbul, Turkey, in 2007. She is currently a Ph.D. student in the Electrical and Electronics Engineering Department at Bilkent University, Ankara, Turkey. 